\title{
Análise das Díades para Compreender a Semelhança da Ação Estratégica: uma aplicação da Regressão Múltipla QAP (MRQAP)
}

\author{
Maurício Reinert ${ }^{1}$ - Universidade Estadual de Maringá - UEM \\ Cristiano de Oliveira Maciel $^{2}$ - Pontifícia Universidade Católica do Paraná - PUC-PR
}

\begin{abstract}
Resumo
O objetivo do artigo é analisar a utilização da Regressão Múltipla via Quadratic Assignment Procedure (QAP) para testar hipóteses sobre a semelhança no comportamento dos atores sociais utilizando medidas diádicas. Destaca-se que os dados empregados na aplicação da técnica figuram como exemplo das potencialidades e dificuldades no tratamento de dados relacionais. Discutem-se os diferentes níveis de análise da Análise de Redes Sociais (ARS) e como o nível da díade pode contribuir para as pesquisas em Ciências Sociais. O procedimento QAP para a avaliação da relação entre matrizes é discutido. Para se analisar a aplicação da técnica é apresentado um exemplo empírico utilizando dados de um survey realizada para obtenção de variáveis relacionais e dados de atributos que caracterizaram a interação entre sessenta pastores de congregações da Assembléia de Deus (AD) em Curitiba, Brasil. O resultado das análises permitiu as seguintes conclusões: (a) as transformações das variáveis é um processo simples, mas requer cuidados, pois parte dos procedimentos é decorrente do julgamento dos pesquisadores, o que pode influenciar o resultado da análise; e (b) por ser uma técnica pouco utilizada e ainda em avaliação quanto a sua robustez, sugere-se parcimônia e consideração dos limites na sua aplicação em investigações sociais.
\end{abstract}

Palavras-chave: QAP, MRQAP, ARS.

\begin{abstract}
The main objective of this article is to analyze the use of Multiple Regression via Quadratic Assignment Procedure (QAP) in order to test the hypothesis about the similarity in the behavior of social actors by using dyadic measurements. The data used in the empirical analysis is just an example of the possibilities and limitations of the test. The different levels of analysis of the Social Network Analysis are discussed as well as how the dyadic level may contribute to the research in the field of Social Sciences. The QAP procedure to evaluate the relationship between matrices is presented as a solution to the dyadic data analysis.
\end{abstract}

\footnotetext{
1 m.reinert@uol.com.br

2 crmaciel.adm@gmail.com
} 
REDES- Revista hispana para el análisis de redes sociales

Vol.22,\#5, Junio 2012

http://revista-redes. rediris.es

In order to analyze the application of the QAO technique, an empiric example is presented in which the data utilized was obtained from: a survey done in order to gather relational variables and from attributes that characterize the standards of interaction among sixty pastors from different Assembléia de Deus (AD) congregations in Curitiba, Brazil. The result of these analyses led to the following conclusions: a) Variable transformation is a simple process, but it requires care and attention, since part of the procedures is a result of the researchers' judgment, and therefore may influence the final result of said analysis; and $b$ ) because it is a technique seldom used and still under evaluation when its strength is considered, parsimony and limit consideration are suggested when applying the technique in social investigation.

Key words: QAP, MRQAP, Social Network Analysis.

\section{Introdução}

A Análise de Redes Sociais (ARS) ganhou impulso ao longo dos últimos 40 anos, principalmente a partir da difusão dos softwares para a sua utilização. No Brasil, o número de trabalhos tem crescido, mas ainda existe muito espaço para o seu desenvolvimento. Autores brasileiros, como Marques (2003; 2010), Kirschbaum (2006), Maciel e Machado-da-Silva (2009), Mello, Crubellate e Rossoni (2010), Verdu (2010) e Verdu e Reinert (2011) são alguns dos que têm utilizado a ARS em suas pesquisas. Todavia, em muitos outros casos, ela tem sido utilizada apenas de maneira descritiva, limitando-se ao mapeamento das redes e identificando características dos atores, tais como centralidade, equivalência estrutural ou buracos estruturais, ou conjuntamente com análises estatísticas tradicionais, transformando as características de redes em atributos dos atores e utilizando-as como variáveis de composição no tratamento dos dados. Entretanto, a alta velocidade na elaboração de novas técnicas para a ARS acentua rapidamente o número de possibilidades de sua aplicação na investigação social.

Entre várias possibilidades de emprego da ARS, a análise das díades ganhou força na última década. Mizruchi e Marquis (2006) propõem que para alguns problemas de pesquisa o foco no nível de análise sociocêntrico (a estrutura de redes como um todo) pode ser substituído, com vantagens, pelo nível de análise da díade, especialmente quando o que se pretende é entender a semelhança do comportamento entre indivíduos. Todavia a utilização desse nível de análise traz desafios, principalmente para o teste de hipóteses. Visto que os tradicionais testes de Regressão Múltipla OLS pressupõem a independência entre as observações, foi preciso encontrar alternativas que solucionassem a frequente violação desse pressuposto na análise das díades. 
REDES- Revista hispana para el análisis de redes sociales

Vol.22,\#5, Junio 2012

http://revista-redes.rediris.es

Entre as soluções para o problema da falta de independência estão a utilização de Efeito Fixo (Fixed Effects) e o Efeito Aleatório (Random Effects). Todavia essas técnicas não funcionam bem para dados diádicos (SIMPSON, 2001).

Uma das soluções que se mostrou mais adequada à análise das díades foi o Quadratic Assignment Procedure (QAP). Essa técnica possibilita a análise da relação entre matrizes quadradas. Segundo Snijders (2011), essa técnica se aplica quando objetivo da pesquisa é controlar para algum efeito específico da rede, por exemplo, o efeito da equivalência estrutural ou de pertencer ao centro ou a periferia da rede. Foi desenvolvida, inicialmente, por Mantel (MANLY, 2008), e aprimorado para a utilização em análises da biologia (LENGENDRE, 2000) e na análise de redes (MIZRUCHI; MARQUIS, 2006; BELL; ZAHEER, 2007). No Brasil, Santos, Rossoni e Machado-da-Silva (2010) utilizaram o QAP para relacionar redes, todavia a análise ficou limitada à correlação e regressão entre redes, sem que outras variáveis diádicas fossem incluídas. Kirschbaum (2011) utilizou o MRQAP para identificar variáveis que influenciem o grau de concordância entre rappers paulistas ao analisarem uma lista de rappers proeminentes.

A partir dessas considerações, o objetivo do artigo é descrever a utilização da Regressão Múltipla QAP Double Dekker semi-partialing para o teste de hipóteses sobre a semelhança no comportamento dos atores sociais, utilizando medidas diádicas de redes e variáveis relacionais. Serão incluídas nos modelos tanto variáveis de redes quanto variáveis diádicas, que medem semelhanças ou diferenças entre os atores da rede. Para a consecução do objetivo foram utilizados os dados de um levantamento (survey) para obtenção de dados relacionais e de composição (dados de atributos) que caracterizaram os padrões de interação entre sessenta pastores de diferentes congregações da Assembleia de Deus (AD), em Curitiba, PR - Brasil. Nesse mesmo levantamento também foram coletadas informações (dados de atributos) sobre quais ações estavam sendo realizadas em cada uma das congregações dirigidas por cada um dos sessenta pesquisados (pastores da AD).

Desse modo, este trabalho visa contribuir com as pesquisas sobre ARS ao apresentar a utilização de uma técnica da análise estatística para variáveis diádicas, cuja utilização ainda não é frequente e pode trazer novas perspectivas para as pesquisas em Ciências Sociais. Dificuldades na operacionalização das análises são discutidas. Essa técnica possibilita ultrapassar a limitação de análise no nível do indivíduo, transformando as 
REDES- Revista hispana para el análisis de redes sociales

Vol.22,\#5, Junio 2012

http://revista-redes. rediris.es

características de rede em atributos dos atores, ou no nível sociocêntrico, o que limita a utilização das técnicas estatísticas tradicionais. Além disso, o nível de análise sociocêntrico requer muitos recursos na sua utilização, pois depende de grandes amostras, sem as quais são limitadas as interpretações (KNOKE; YANG, 2008).

\section{Diferentes Concepções para a Análise de Redes Sociais (ARS)}

O termo Análise de Redes Sociais (Social Network Analysis) é frequentemente classificado como: (i) metáfora; (ii) teoria; e, (iii) método (POWELL; SMITH-DOERR, 1994). Enquanto metáfora, as redes sociais são representadas pelo conceito mais simples e elástico de conjunto de conexões entre determinados elementos. Como teoria, especificamente na área de ciências sociais, quase toda explicação que revele um conteúdo informacional associado ao fenômeno de imersão social (embeddedness) é descrita como "análise" de redes (entenda-se o termo análise como perspectiva) (DACIN; VENTRESCA; BEAL, 1999; BORGATTI; FOSTER, 2003). Quando classificado como método, a Análise de Redes Sociais se caracteriza não centralmente como uma estratégia de pesquisa (e.g., survey, etnografia, estudo de caso), mas, sobretudo, como um conjunto de técnicas empregadas particularmente no tratamento de dados relacionais (variáveis de laços entre atores sociais) de diferentes níveis, uma vez que essas técnicas podem ser empregadas em vários delineamentos.

O foco do trabalho é a Análise de Redes Sociais como "método". Ela é mais apropriadamente descrita como um conjunto de técnicas de tratamento de dados sobre laços ou conexões entre agentes (nós ou vértices), ou seja, dados relacionais. Enquanto as variáveis convencionais, como idade, altura e respostas a questões com escala de Likert são exemplos bem conhecidos de variáveis de atributos ou de composição, variáveis relacionais ou estruturais são frequentemente binárias, representam a existência ou não de relação entre dois atores sociais e, normalmente, obedecem a uma disposição matricial para o exercício de tratamento de dados (WASSERMAN; FAUST, 1994).

Em função dessas características, uma matriz de dados relacionais está sujeita ao emprego de diferentes técnicas. Essas técnicas são escolhidas em função dos argumentos teóricos e dos níveis de análise considerados pelo pesquisador. Esses níveis possibilitam tratar as redes sociais, como: (i) egocêntricas; (ii) sociocêntricas; e (iii) diádicas. Medidas egocêntricas contemplam as características estruturais de cada indivíduo, medidas sociocêntricas consideram as características da rede como um todo 
REDES- Revista hispana para el análisis de redes sociales

Vol.22,\#5, Junio 2012

http://revista-redes. rediris.es

e as medidas diádicas representam características de pares de atores sociais que estão dentro de uma rede (MIZRUCHI; MARQUIS, 2006).

Esses diferentes níveis de análise são resultados da evolução da ARS na psicologia social, na antropologia social e na sociologia. De acordo com Scott (2000, p.7), diversas "ondas" podem ser identificadas no desenvolvimento da Análise de Redes Sociais e na constituição de seus níveis de investigação. De acordo com ele, essas "ondas" apresentam intersecções "umas com as outras em uma história complexa e fascinante, às vezes convergindo e outras divergindo em cada um de seus caminhos particulares" (SCOTT, 2000, p. 7). Entre suas divergências foi marcante a diferença de ênfase em abordagens egocêntricas, sociocêntricas e diádicas.

As medidas egocêntricas enfatizam as relações em torno dos indivíduos (nós) da rede (e.g., ZAHEER; BELL, 2005; AHUJA, 2000). Assim, as inferências dos estudos que se enquadram nessa abordagem são dirigidas aos indivíduos. Desse modo, cada ator social tem algumas de suas medidas egocêntricas consideradas para que tais medidas também possam ser associadas às variáveis de resultado, normalmente padrões comportamentais. Entre as medidas de ego mais simples e frequentemente utilizadas estão as centralidades de grau (degree), proximidade (closeness) e intermediação (betweenness) (HANNEMAN, 2001). Entre as características estruturais de ego mais elaboradas e amplamente empregadas estão as medidas de buracos estruturais (structural holes), desenvolvidas por Burt (1992; 2004).

As medidas sociocêntricas, por outro lado, são extraídas ao considerar a totalidade do conjunto de relações que dão forma a uma rede social. Portanto, sua ênfase recai na análise de características da rede como um todo ou de seus subgrupos constituintes. Nos estudos que adotam essa abordagem tem sido comum a quantificação dos relacionamentos entre agentes no interior de grupos previamente definidos para explicar como padrões de interação estão associados a alguns tipos de resultados, frequentemente comportamentais. De acordo com Scott (2000), em trabalhos com esse objetivo, a densidade da rede ou dos grupos (proporção de conexões, entre nós ou vértices, comparada ao número máximo possível de laços em uma rede) tem sido uma das características consideradas mais frequentemente. As medidas de centralização da rede (diferente de centralidade dos nós) e a análise centro-periferia também são exemplos das técnicas empregadas nessa abordagem (BORGATTI; EVERETT, 1999). 
REDES- Revista hispana para el análisis de redes sociales

Vol.22,\#5, Junio 2012

http://revista-redes.rediris.es

As medidas diádicas, por sua vez, representam a relação entre dois atores na rede (WASSERMAN; FAUST, 1994; KNOKE; YANG, 2008). Elas não são um atributo de um ator isolado, pois existe somente enquanto os atores mantiverem a relação, ou seja, é uma propriedade conjunta dos atores (KNOKE; YANG, 2008). Entre essas medidas estão as de distância entre os atores na rede, por exemplo, distância geodésica, o menor caminho que liga dois atores na rede, e medidas de posição, como, por exemplo, a equivalência estrutural, a qual indica se dois atores ocupam posições equivalentes na redes, ou seja, em que medida mantém ligação com os mesmos atores (SCOTT, 2000).

As medidas diádicas são interessantes, uma vez que representam um nível de análise supra-individual, possibilitando, assim, a análise de variáveis relacionais, nas quais o objetivo não é compreender o comportamento isolado de um indivíduo, mas sim o seu comportamento em relação a outro indivíduo. A relação entre tais medidas e a semelhança de comportamento é explorada na próxima subseção do artigo.

\section{ARS e o Exame da Semelhança de Comportamento}

Analisar comportamentos semelhantes não é uma novidade nas pesquisas das Ciências Sociais (MURNIEKS, et al., 2011), entretanto a sua utilização tem aumentado nos últimos anos em conjunto com a expansão da ARS (MIZRUCHI; MARQUIS, 2006) e com o interesse em compreender a atuação conjunta entre organizações, tais como as Alianças Estratégicas e a cooperação com fornecedores. Mizruchi e Marquis (2006) afirmam que, em pelo menos três situações, o nível de análise da díade é apropriado: (a) quando o pesquisador está interessado nas transações entre agentes; (b) quando o objetivo é analisar a similaridade de comportamento entre atores, e esse comportamento é medido quantitativamente; e (c) quando o objetivo é analisar a semelhança de comportamento entre uma série de eventos discretos.

Para que seja possível analisar a semelhança do comportamento, todas as variáveis precisam estar no mesmo nível de análise, ou seja, precisam ser relacionais. Muitas das medidas de redes são diádicas, entretanto as variáveis normalmente utilizadas nas análises estatísticas nas Ciências Sociais representam atributos do indivíduo. Faz-se necessário, portanto, a transformação das variáveis, pois estas precisam indicar em que medida dois atores são semelhantes em relação aos atributos analisados ou que participam de eventos simultâneos ou categorias semelhantes. Essa transformação é possível tanto para variáveis discretas quanto para variáveis contínuas. 
REDES- Revista hispana para el análisis de redes sociales

Vol.22,\#5, Junio 2012

http://revista-redes. rediris.es

Para as variáveis discretas, é possível utilizar a co-presença como indicador e que dois atores participam de um mesmo evento ou fazem parte da mesma categoria. Cria-se uma variável dummy, na qual um dos valores indica que os atores estão co-presentes em um evento ou fazem parte da mesma categoria e o outro indica que não. Além disso, podem ser utilizadas medidas de semelhança quando é considerado um grupo de variáveis dicotômicas. Tais medidas são definidas como Empate Simples (Simple Match), Índice de Ochiai e Índice de Jaccard. Todas essas medidas variam de 0 a 1 , na qual o 0 indica nenhuma similaridade e o 1 similaridade completa (MANLY, 2008).

As variáveis contínuas podem ser transformadas, utilizando-se a Distância Euclidiana. Ela é simplesmente o somatório das diferenças ao quadrado entre os escores das variáveis que estão sendo utilizadas na análise (MANLY, 2008). Essa medida é utilizada para a Análise de Cluster Hierárquica na estatística (CRAMER, 2003) e para a Equivalência Estrutural na ARS (KNOKE; YANG, 2008). Ela indica a diferença entre dois atores em relação à determinada variável ou variáveis. Pode ser padronizada para que as diferenças de mensuração não afetem os resultados com relação à escala de mensuração e ao número de variáveis que estão sendo utilizadas para o cálculo da diferença (MANLY, 2008). Para facilitar a comparação entre as variáveis dicotômicas e as contínuas, o SPSS possui uma ferramenta de padronização da Distância Euclidiana, na qual os valores da distância saem padronizados de 0 a 1, sendo 0 a menor distância e 1 a maior distância (SPSS, 2009). A Distância Euclidiana é exatamente o oposto da semelhança entre dois atores, por isso seu inverso pode ser utilizado como medida de semelhança.

Um dos maiores desafios para a análise de medidas diádicas é o problema da independência das observações. As técnicas estatísticas tradicionais, sejam elas bivariadas ou multivariadas, possuem como pressuposto que as observações sejam independentes entre si (COHEN et al., 2003). A definição de díade implica no ferimento desse pressuposto, pois a díade é formada por um par de atores, e um mesmo ator pode estar presente em diversas díades. E no caso da análise de semelhança entre atores, cada ator participa de $(\mathrm{N}-1)$ díades (sendo $\mathrm{N}$ o número de atores participantes da pesquisa), ou seja, serão colocadas como observações as relações desse ator com todos os demais atores da análise, excluindo apenas a relação dele com ele mesmo. Além disso, cada um dos atores participará do mesmo número de díades. O número total de observações em uma amostra de 50 atores será de 1225 
REDES- Revista hispana para el análisis de redes sociales

Vol.22,\#5, Junio 2012

http://revista-redes. rediris.es

$(\mathrm{N}(\mathrm{N}-1) / 2)$, se a rede for não-direcional, e de $2450(\mathrm{~N}(\mathrm{~N}-1)$ se for tratada como direcional, visto que cada díade corresponde a uma observação.

A falta de independência entre as observações pode levar à subestimação do erro de Tipo I. Existem algumas técnicas para lidar com esse problema. Dentre essas técnicas estão o Efeito Fixo (Fixed Effects) e o Efeito Aleatório (Random Effects). Entretanto, nenhum das duas técnicas funciona bem no caso de dados diádicos (SIMPSON, 2001).

Para a análise de redes e de semelhança entre comportamento, a técnica mais apropriada é o QAP - Quadratic Assignment Procedure. Essa técnica utiliza a Regressão Linear (OLS), todavia ajusta o coeficiente individual pela probabilidade dele ser maior ou menor que o coeficiente que ocorreria ao acaso (MIZRUCHI; MARQUIS, 2006). Esse procedimento foi desenvolvido por Mantel na década de 60 para compreender se casos de uma doença que ocorre próxima no espaço também tende a estar próxima no tempo (MANLY, 2008). A técnica QAP avalia se duas matrizes quadradas são significativamente relacionadas (KRACKHARDT, 1987). Este é um procedimento que testa se o resultado da correlação é espúrio por meio da repetição da permutação entre linhas e colunas de uma das matrizes e novo cálculo da correlação ou regressão. Gera-se uma distribuição de valores aleatórios, a qual é comparada com o resultado originalmente observado, e um nível de significância pode ser inferido. Enquanto a estatística descritiva é gerada pela OLS, a probabilidade inferencial é gerada de maneira não-paramétrica. Se o teste QAP de similaridade entre as matrizes apresentar uma baixa probabilidade significativa, pode-se então concluir que a correlação não é espúria (KRACKHARDT, 1987).

Krackhardt (1988) propõe a extensão do QAP para o modelo de regressão múltipla, e encontram bons resultados, sugerindo que o procedimento é apropriado, dentro de determinados limites, principalmente o número de variáveis independentes e a assimetria dos dados.

Devido ao interesse que tem gerado, especialmente para o tratamento de dados de Redes Sociais, o QAP tem sido aprimorado. Dekker, Krackhardt e Snijders (2007) apresentam um novo método de permutação a ser utilizado no QAP de Regressão Múltipla, o Double Semi-Partialling (DSP) ${ }^{3}$. Os testes realizados demonstram que esse novo método é mais robusto que os anteriores, e que esse é o teste mais seguro para

\footnotetext{
${ }^{3}$ Para o detalhamento do teste verificar Dekker, Krackhardt e Snijders (2007).
} 
REDES- Revista hispana para el análisis de redes sociales

Vol.22,\#5, Junio 2012

http://revista-redes. rediris.es

ser utilizado na relação entre variáveis diádicas. Esse teste está disponível no pacote de ARS UCINET 6.247.

Em virtude da discussão realizada, é exemplificada a utilização da técnica MRQAP para o teste de hipóteses da relação entre dados diádicos. O delineamento do estudo para coleta dos dados diádicos está descrito na próxima seção.

\section{Exemplo Empírico}

Apresenta-se agora um exemplo empírico descrevendo o passo a passo para a construção do modelo, desde a transformação das variáveis até a aplicação da técnica e a análise dos resultados. Por tratar-se de um exemplo da aplicação do método, o foco está no tratamento e análise dos dados e não nos resultados. O delineamento do estudo para coleta dos dados diádicos está descrito na próxima seção.

\section{Procedimento metodológico}

Os dados aqui submetidos ao QAP - Quadratic Assignment Procedure - foram gerados em um estudo que teve como objetivo avaliar a relação entre valores dos pastores dirigentes de sessenta congregações da Igreja Assembléia de Deus (AD), em Curitiba, a rede de relacionamentos entre esses pastores e as atividades estratégicas desenvolvidas pelas congregações. Foram coletados dados para identificar quais valores ambientais eram mais importantes, quais os dirigentes com que cada um (pastor ou evangelista da congregação) mantinha relacionamento mais frequentemente e quais atividades estratégicas eram empregadas. Apenas o dirigente principal (pastor ou evangelista) de cada congregação respondeu o questionário e figurou como o nó da rede. Portanto a rede não foi tratada como multiplex. Os dados foram coletados por meio de questionário. Os dados dessa pesquisa se encaixam perfeitamente na situação descrita por Mizruchi e Marquis (2006), em que se busca compreender a semelhança de comportamento, no caso a realização de determinadas ações estratégicas, as quais são medidas de maneira quantitativa.

Para identificar quais seriam os valores relacionados à gestão, que poderiam ser percebidos pelos pastores como mais ou menos importantes, foi feito um estudo de 2028 arquivos que agregou três anos de publicações de revistas e jornais impressos (MACIEL, 2007). Para reduzir a probabilidade de viés de um ou outro tipo de publicação foram consideradas várias fontes de informação, como: site da SEPAL (Serviço de Evangelização Para a América Latina), Jornal Folha de São Paulo, Jornal 
REDES- Revista hispana para el análisis de redes sociales

Vol.22,\#5, Junio 2012

http://revista-redes. rediris.es

Gazeta do Povo, Revista Veja, Revista Exame, artigos de periódicos científicos, livros e reportagens do programa de televisão Jornal Nacional. A delimitação desses documentos ocorreu por meio da busca por palavras-chave (i.e., evangélicos e católicos). O tratamento dos dados qualitativos ocorreu por Análise Temática, tendo o texto completo como unidade de significação, conforme sugerido por Bardin (2009). Como resultado desse procedimento foi identificado inicialmente um conjunto de vinte temas. Mas após uma avaliação das similaridades entre os temas alguns elementos foram agregados e outros descartados porque não pertenciam a esfera dos valores relacionados à gestão.

O resultado final da análise desses documentos permitiu delimitar 12 valores ambientais principais que estavam presentes de modo mais acentuado no campo das organizações religiosas. Esses valores são: inserção política; expansão da igreja; responsabilidade social; modernidade; eficiência da gestão; customização para os fiéis; qualidade dos cultos e templos; reputação; tradição; cooperação interorganizacional entre congregações; competição por fiéis; e ênfase no mercado cristão. Após a delimitação e definição desses valores, foram construídos três indicadores em escala tipo Likert, fazendo referência à importância de cada valor ambiental. Os doze valores foram mensurados por meio do questionário com escala de múltiplos indicadores. Os indicadores de cada fator constam no apêndice.

Para mapear a rede de relacionamentos, o questionário continha um campo específico em que era solicitado ao respondente que indicasse de três a seis nomes de outros dirigentes da Assembleia de Deus com quem ele mantinha contato mais frequente. $O$ número resultou das análises das entrevistas com pastores na qual uma pergunta aberta sobre com quais outros dirigentes eles mantinham contato mais frequente e as respostas não ultrapassaram a indicação de seis nomes. A questão para geração dos dados relacionais também consta no apêndice.

Para identificar as atividades estratégicas realizadas pelas congregações foram feitas 7 entrevistas com pastores. Materiais impressos, como folhetos e documentos da igreja, também foram coletados com esses pastores. Essa etapa permitiu verificar quais eram as atividades empregadas com maior frequência pelos pastores na gestão de sua congregação. Foram identificadas 36 atividades, que incluíam doações, reuniões, visitas aos fiéis e diversos tipos de cultos. Os respondentes assinalavam quantas 
REDES- Revista hispana para el análisis de redes sociales

Vol.22,\#5, Junio 2012

http://revista-redes.rediris.es

atividades desejassem. Resultavam, então, 36 variáveis categóricas nas quais 0 indica a não realização da atividade e 1 , a sua realização.

O questionário final continha, então: 36 variáveis em 12 blocos, conforme os valores ambientais, indicação dos nomes dos pastores que cada entrevistado possuía contato mais frequente, mais a descrição de 36 atividades estratégicas (extraídas das entrevistas e documentos) para as quais o entrevistado assinalava se aquela atividade era presente em sua congregação ou não e perguntas sobre a idade e posição na hierarquia da igreja. Em relação à hierarquia na igreja, alguns dos dirigentes eram pastores e outros apenas evangelistas. Essa variável categórica foi utilizada como representativa do status de cada dirigente. A importância dos valores foi assinalada em uma escala de importância de 1 a 10 (escala intervalar tipo Likert). Essas variáveis tiveram suas consistências internas avaliadas por meio do Alfa de Cronbach, todas apresentaram índices acima de 0,80.

\section{Análise dos dados}

A análise dos dados consistiu em duas etapas. Na primeira, foi examinada visualmente a matriz e o sociograma da rede. Na segunda etapa ocorreu a análise da semelhança das ações na Rede da Assembleia de Deus (AD).

Para verificar a necessidade e possibilidade da análise de semelhança de comportamento dos nós (agentes) da rede, foi inicialmente observada a configuração do padrão de relacionamentos dos dirigentes pesquisados. Para tanto, os dados coletados via survey foram inseridos em uma matriz no software Ucinet e, posteriormente, foi gerado o sociograma no programa Pajek (Figura 1). Conforme o sociograma, os relacionamentos entre os atores da rede se mostraram bastante fluídos $^{4}$, sem presença clara de grupos ou componentes principais, o que torna particularmente útil a análise de centro-periferia (BORGATTI; EVERETT, 1999). Esta análise agrupa os casos mais homogêneos, o centro, e classifica os casos isolados como periferia (BORGATTI; EVERETT, 1999). Na rede da Assembleia de Deus, o centro é composto por 28 dirigentes (Figura 1), cuja representação se faz pelos nós na cor amarela e a periferia é composta por 32 dirigentes, nós na cor verde.

\footnotetext{
${ }^{4}$ Foi realizada uma análise de cluster para a identificação de agrupamentos, entretanto o dendrograma não mostrou grupos claramente distintos, e a análise de homogeneidade apresentou índice E-I (KRACKHARD e STERN, 1988) para dois grupos foi de 0,344, o que sugere pouca homogeneidade interna, ou seja, a não existência de agrupamentos bem definidos.
} 
REDES- Revista hispana para el análisis de redes sociales

Vol.22,\#5, Junio 2012

http://revista-redes.rediris.es

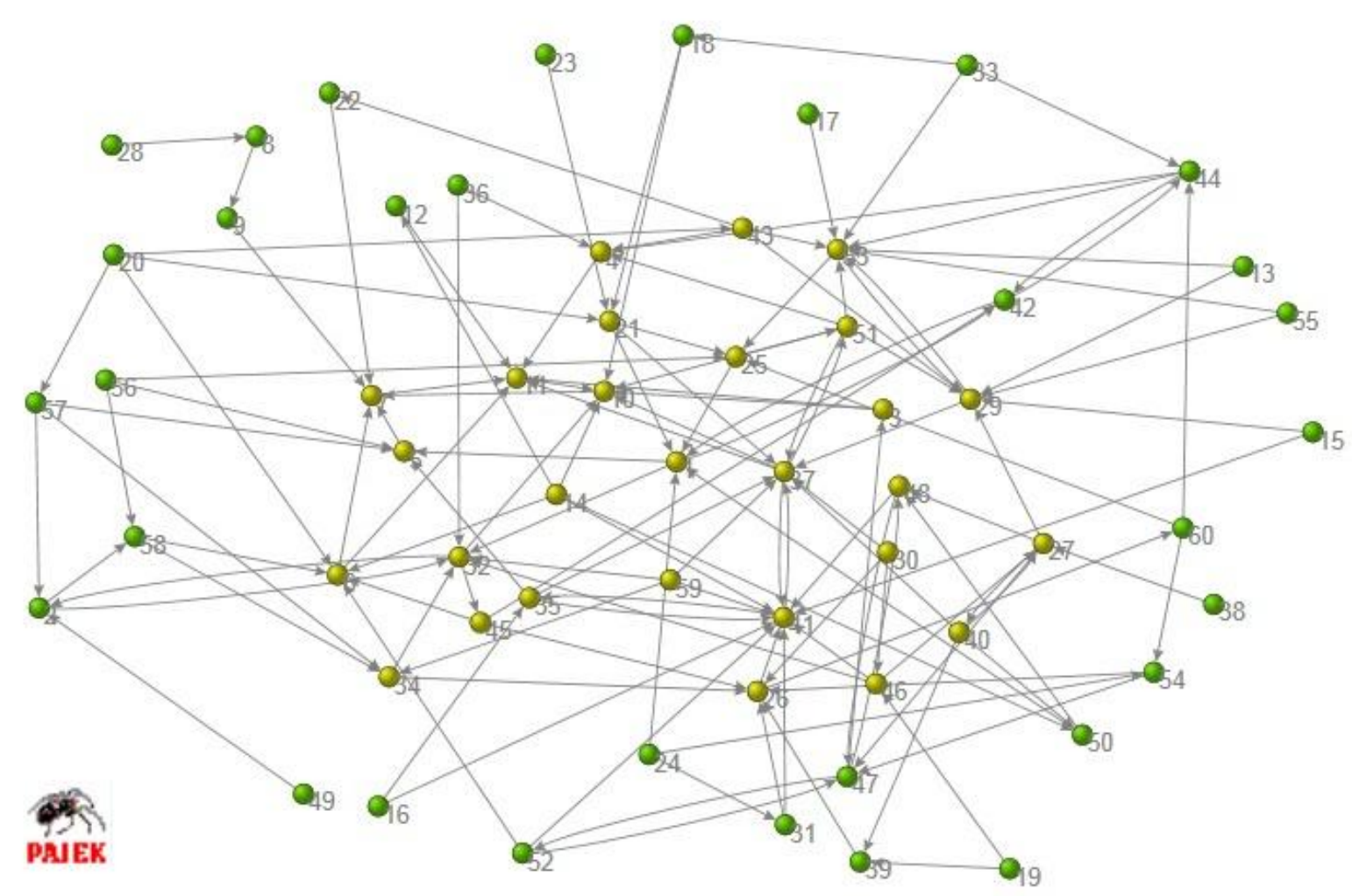

Figura 1. Sociograma centro-periferia da rede. Fonte: dados primários.

As dificuldades que seriam impostas pelo tratamento da rede como arranjo sociocêntrico e dos atores individuais com variáveis de atributo contribuíam para aplicação da análise no nível das díades, pois essa forma alternativa de tratamento dos dados possibilita escapar de violações nos pressupostos dos testes estatísticos, em função da amostra de apenas 60 casos para teste de hipóteses sobre propriedades das redes e dos nós e seu comportamento (i.e., atividades estratégicas).

A análise da semelhança, entre ações, propriedades da rede e outros atributos no nível da díade, ocorreu da seguinte maneira: a partir das variáveis de atributos coletados no estudo citado anteriormente, fez-se a transformação dessas em variáveis diádicas. A primeira variável dicotômica a ser criada foi a variável dependente Semelhança das Ações Estratégicas (SAE). Originalmente foram identificadas e pesquisadas 36 Ações, as quais foram mensuradas de maneira discreta - presença ou ausência das Ações. Entretanto, das 36 ações, algumas foram apontadas apenas por poucos respondentes e outras por quase todos eles.

Nos casos extremos, Campanhas de Arrecadação Financeira e Busca de Doações com Empresários foram apontadas por apenas dois respondentes, e Visitas para os 
REDES- Revista hispana para el análisis de redes sociales

Vol.22,\#5, Junio 2012

http://revista-redes.rediris.es

Ausentes nos Cultos foram apontadas por 58 respondentes. Optou-se, então, por eliminar os casos em que acontecia pouca variação nas ações estratégicas. O objetivo era eliminar aquelas ações que eram realizadas por muitos ou poucos dos respondentes, e que por isso não são sensíveis às influências das variáveis independentes. Como não existe um critério ótimo para fazê-lo, optou-se por eliminar aquelas que eram realizadas por menos de $25 \%$ ou por mais de $75 \%$ dos respondentes, ou seja, cuja freqüência absoluta era menor que 15 ou maior que 45. Esse procedimento fez com que apenas as ações estratégicas com maior variação de frequência permanecessem na análise. As ações estratégicas restantes são apresentadas na tabela 1. Para a criação da variável SAE utilizou-se a técnica do Empate Simples (Simple Match) já descrito anteriormente (MANLY, 2008). O procedimento foi realizado utilizando o SPSS 18. A variável resultante vai de 0 (completa semelhança) a 1 (completa diferença).

\begin{tabular}{lcc}
\hline \multicolumn{1}{c}{ Atividade Estratégica } & Freqüência Absoluta & Percentual \\
\hline Culto da vitória & 23 & $38,30 \%$ \\
Culto ao ar livre & 42 & $70,00 \%$ \\
Envio de missionários no exterior & 29 & $48,30 \%$ \\
Envio de missionários em outros & 34 & $56,70 \%$ \\
estados & & \\
Curso de música & 42 & $70,00 \%$ \\
Plantão voluntário na central & 17 & $28,30 \%$ \\
Uso de e-mail na evangelização & 25 & $41,70 \%$ \\
Uso de internet na evangelização & 25 & $41,70 \%$ \\
Reformas na congregação & 38 & $63,30 \%$ \\
Evangelização nas ruas à noite & 22 & $36,70 \%$ \\
Planejamento e realização de retiros & 29 & $48,30 \%$ \\
Intensos estímulos individuais para & 20 & $33,30 \%$ \\
batismo & & $46,70 \%$ \\
Doações de alimentos & 28 & $51,70 \%$ \\
Divulgação no jornal da AD & 31 & \\
Fonte: dados primários. & &
\end{tabular}

Tabela 1: Frequência da presença das atividades estratégicas.

A segunda variável criada foi a Diferença de Idade (DI). A idade foi medida em anos e a sua transformação em variável diádica foi feita por meio da técnica da Distância Euclidiana. Novamente utilizando-se do SPSS 18, calculou-se a Distância Euclidiana, re-escalonando para um intervalo de 0 (completa semelhança) e 1 (completa diferença). É importante salientar que diferente da variável anterior que media a semelhança, essa mede a diferença, todavia elas são apenas opostas, ou seja, é possível transformar a diferença em semelhança fazendo-se a inversão dos valores. 
REDES- Revista hispana para el análisis de redes sociales

Vol.22,\#5, Junio 2012

http://revista-redes.rediris.es

A variável Semelhança de Status foi criada a partir da lógica da co-presença, ou seja, quando os dois respondentes pertenciam à mesma categoria, ambos eram evangelistas ou ambos eram pastores, então era atribuído o valor 1 , e quando eles pertenciam a categorias diferentes, era atribuído valor 0 . A transformação foi feita por meio do SPSS 18, utilizando a análise de distâncias técnica de Empate Simples (Simple Match).

Por fim, foram criadas 12 variáveis com as Diferenças de Valores (DV). Como as variáveis dos valores eram escalares, utilizou-se a Distância Euclidiana para realizar a transformação. Novamente utilizando-se o SPSS 18 para o cálculo da Distância Euclidiana re-escalonada para um intervalo de 0 a 1. a tabela 2 apresenta as variáveis de diferenças entre os valores.

\begin{tabular}{ll}
\hline & Valores \\
\hline DV01 - Inserção Política \\
DV02 - Expansão \\
DV03 - Responsabilidade Social \\
DV04 - Modernidade \\
DV05 - Eficiência (Gestão) \\
DV06 - Customização \\
DV07 - Qualidade \\
DV08 - Reputação \\
DV09 - Tradição \\
DV10 - Cooperação \\
DV11 - Competição \\
DV12 - Mercado
\end{tabular}

Tabela 2: Variáveis de Diferença de Valores. Fonte: dados primários.

A variável das redes Semelhança no Posicionamento na Estrutura da Rede (SP) foi criada a partir da análise centro-periferia utilizando a co-presença, ou seja, quando ambos na díade pertenciam ao mesmo grupo era caracterizada a semelhança e atribuído valor 1 ; do contrário era caracterizada a diferença e atribuído o valor 0 . A transformação foi feita por meio do SPSS 18, utilizando a análise de distâncias técnica de Empate Simples (Simple Match).

A partir daí, surgiram 15 matrizes quadradas contendo as diferenças ou semelhanças dos participantes da rede pesquisada em relação às variáveis a serem testadas na análise, sendo as duas primeiras variáveis de controle, a diferença de idade (DI) e semelhança na hierarquia da igreja (SS), as 12 diferenças de valores (DV) e, por fim, a característica estrutural da rede a partir da análise centro-periferia (SP). São essas matrizes que serão utilizadas para a análise de regressão múltipla pelo QAP - Double Dekker Semi-Partialing. 
REDES- Revista hispana para el análisis de redes sociales

Vol.22,\#5, Junio 2012

http://revista-redes. rediris.es

As hipóteses a serem testadas relacionam a diferença de valores e a semelhança de posição na estrutura da rede às ações estratégicas, ou seja, a diferença de valores está negativamente associada à realização das ações estratégicas (H1), enquanto a semelhança na posição na rede está positivamente associada às ações estratégicas (H2). A primeira hipótese decorre da noção bastante utilizada na psicologia social de que a similaridade entre indivíduos leva a tomarem decisões parecidas (MURNIEKS et al., 2011), sendo que o oposto é verdadeiro. No exemplo empírico, a diferença entre os valores dos indivíduos se relacionaria negativamente com Semelhança nas Ações Estratégicas. A segunda hipótese decorre da noção de que grupos estruturalmente homogêneos tendem a agir de maneira semelhante (FELD, 1982). No exemplo empírico, a análise centro-periferia apresentou um grupo homogêneo posicionado no centro, com indivíduos isolados ao redor. A hipótese é de que indivíduos posicionados de maneira semelhante no grupo agem de maneira semelhante.

A tabela 3 apresenta os três modelos construídos. O primeiro é o modelo base em que foram inseridas apenas as variáveis de controle. No segundo modelo as variáveis das diferenças de valores são acrescentadas. Finalmente, no terceiro modelo, foi incluída a variável de semelhança na posição na rede.

\begin{tabular}{llcl}
\hline & Modelo 1 & Modelo 2 & Modelo 3 \\
\hline Intercepto & & 0,6928 & 0,6789 \\
Diferença de Idade & $-0,1972 * *$ & $-0,1117 *$ & $-0,0908$ \\
Semelhança de Status & $-0,0001$ & 0,0013 & 0,0006 \\
DV1 - Inserção Política & & 0,0067 & 0,0059 \\
DV2 - Expansão & & $-0,1128^{* *}$ & $-0,1140 * *$ \\
DV3 - Responsabilidade Social & & $-0,1247 * *$ & $-0,1257^{* *}$ \\
DV4 - Modernidade & $-0,0708^{* *}$ & $-0,0719^{* *}$ \\
DV5 - Eficiência (Gestão) & & $-0,0398$ & $-0,0350$ \\
DV6 - Customização & $-0,0221$ & $-0,0230$ \\
DV7 - Qualidade & $-0,0509 *$ & $-0,0484^{*}$ \\
DV8 - Reputação & & $-0,1459 * *$ & $-0,1435^{* *}$ \\
DV9 - Tradição & $-0,0103$ & $-0,0088$ \\
DV10 - Cooperação & 0,0256 & $-0,0235$ \\
DV11 - Competição & & $-0,0057$ & $-0,0069$ \\
DV12 - Mercado & & $-0,0683 * *$ & $-0,0689 * *$ \\
Semelhança de Posicionamento & & & $0,0242^{* *}$ \\
\hline$R^{2}$ & 0,021 & 0,191 & 0,196 \\
$R^{2}$ Ajustado & 0,020 & 0,188 & 0,192 \\
Probabilidade & 0,000 & 0,000 & 0,000 \\
No de observações & 3540 & 3540 & 3540 \\
\hline
\end{tabular}

Tabela 3: Modelos de Regressão Múltipla QAP (DSP) - variável dependente SAE. Fonte: dados primários

$$
\text { * }(p \text {-value }<0,05) ; * *(p \text {-value }<0,01)
$$


REDES- Revista hispana para el análisis de redes sociales

Vol.22,\#5, Junio 2012

http://revista-redes.rediris.es

Os resultados da tabela 3 indicam que a maioria das variáveis seguiu a direção esperada no Modelo 3, ou seja, as variáveis que medem semelhança têm seus betas com sinal positivo e as que medem diferença têm seus betas com sinal negativo, visto que a variável dependente é a semelhança entre as atividades estratégicas realizadas. A única exceção foi a variável DV1 - Inserção Política que possui beta positivo, todavia não apresentou significância estatística. No Modelo 1 a única variável que apresentou significância estatística foi a Diferença de Idade (DI), todavia o $\mathrm{R}^{2}$ foi de apenas 0,021 , o que indica que o modelo explica apenas $2 \%$ da variável dependente.

A introdução das variáveis de diferença de valores no Modelo 2 alterou bastante os resultados, elevando o $\mathrm{R}^{2}$ para 0,191. A Diferença de Idade continuou significativa, mas agora a 95\%, e com diminuição do valor do beta, mas esse manteve sua relação negativa com a variável dependente. A variável Semelhança de Status teve o sinal do beta alterado assumindo relação positiva com a variável SAE, conforme o esperado, mas mantendo a não significância estatística. Entre as variáveis de diferença de valores, o valor Qualidade (DV7) mostrou-se estatisticamente significativo a 95\%, e as variáveis Expansão (DV2), Responsabilidade Social (DV3), Modernidade (DV4), Reputação (DV8) e Mercado (DV12) apresentaram significância estatística a 99\%, todas com o sinal do beta negativos como o esperado pela teoria, corroborando $\mathrm{H} 1$.

Finalmente, no Modelo 3 foi introduzida a variável Semelhança de Posicionamento na Rede. O $\mathrm{R}^{2}$ teve um aumento de 0,05 e foi para 0,196, melhorando o modelo. A variável Diferença de Idade não se apresentou mais estatisticamente significativa, mas as variáveis de diferença de valor mantiveram-se significativas. A variável Cooperação (DV10) que aparecia com o sinal invertido no Modelo 2 aparece agora com o sinal igual o esperado; apenas a variável Inserção Política manteve-se com o sinal inverso ao esperado. A variável Semelhança de Posicionamento na Rede é significativa a 99\%, corroborando $\mathrm{H} 2$.

A probabilidade indicada na penúltima linha evidencia a proporção das permutações aleatórias que possuíam $\mathrm{R}^{2}$ mais altos do que o do observado no modelo. Quanto mais baixa essa probabilidade, menor é a chance do resultado ser espúrio, o que indica o bom ajuste do modelo. É importante destacar ainda a última linha da tabela 2 que apresenta o número de observações utilizadas na Regressão Múltipla QAP. Ele é o número de díades formadas pelos 60 participantes da rede. Além disso, cabe enfatizar 
REDES- Revista hispana para el análisis de redes sociales

Vol.22,\#5, Junio 2012

http://revista-redes.rediris.es

que os modelos foram rodados utilizando o valor padrão de 2.000 permutações no QAP.

A partir dessas análises, a discussão dos resultados será direcionada à possibilidade de utilização da Regressão Múltipla QAP (DSP) nas pesquisas em Ciências Sociais, utilizando a ARS.

\section{Discussão dos Resultados da Aplicação do MRQAP}

Nas pesquisas quantitativas sobre Redes tem-se como um dos aspectos mais importantes deslocar da descrição dos dados para a inferência estatística. Não que a descrição não seja importante, mas não se deve contentar apenas com ela, pois se existe um interesse efetivo na geração de conhecimento é preciso dar um passo adiante nas análises. É o que está ocorrendo agora com a ARS. Se, em um primeiro momento, a imagem dos mapas das redes era suficiente para impressionar e trazer uma nova perspectiva para problemas de pesquisa na área, entra-se agora em um momento em que análises mais complexas são necessárias.

O nível de análise da díade pode trazer um novo olhar para antigos problemas de pesquisa. A sua utilização é possível em áreas tão diferentes, tais como Sociologia Econômica, Teoria das Organizações e Administração, entre outras. O que é fundamental é que o problema de pesquisa envolva a similaridade ou a dissimilaridade de comportamento dos atores envolvidos.

O nível de análise deve ser o do relacionamento entre os nós pesquisados, a díade, sejam eles pessoas, organizações, ou quaisquer outros tipos de observações. Os dados das díades podem ser obtidos por meio da transformação de dados de atributo para dados relacionais, utilizando as técnicas discutidas anteriormente e disponíveis nos pacotes estatísticos largamente utilizados em Ciências Sociais. As transformações são possíveis tanto para variáveis discretas quanto para contínuas. O cuidado que é preciso ter é o de manter uma coerência entre a variável transformada e a teoria que se está utilizando na construção da hipótese. No exemplo apresentado, as hipóteses a serem testadas apresentavam uma fundamentação teórica coerente, ou seja, a teoria previa uma semelhança de comportamento a partir de uma semelhança de valores (MURNIEKS et al., 2011), e uma semelhança do posicionamento na rede (FELD, 1882). 
REDES- Revista hispana para el análisis de redes sociales

Vol.22,\#5, Junio 2012

http://revista-redes. rediris.es

Sem a sustentação teórica, como qualquer outra análise estatística, a QAP perde sentido.

Além do cuidado com a coerência teórica, a construção das variáveis precisa ser pautada pela parcimônia no momento de decisão de juntar o que pode ser considerado semelhante. As técnicas para medir distâncias multivariadas utilizadas nas transformações são caracterizadas como descritivas e não como inferenciais, assim como a Análise de Agrupamentos (HAIR, 2005). Elas possuem uma fundamentação matemática, mas não estatística, ou seja, não existe um critério a priori apropriado para a decisão de quantas variáveis e quais devem ser agrupadas.

Nas análises realizadas para esse trabalho foram identificadas algumas dificuldades que podem ser enfrentadas na transformação dos dados de atributos para o de díades. Quando as medidas de distância são de uma variável só, tais como a idade ou o status utilizados no exemplo empírico, não existe problema algum; o único cuidado é a padronização das variáveis para evitar que a escala em que elas são medidas influencie os resultados das análises. Entretanto, as variáveis Diferenças de Valores poderiam ser agrupadas em uma variável apenas, utilizando a Distância Euclidiana das 12 em conjunto. Isso faria sentido teoricamente imaginando que a variável final indicaria uma diferença geral dos 12 valores entre dois atores. O problema é que a análise fatorial realizada antes identificou que cada um desses valores faz parte de uma dimensão diferente, o que causaria problema ao tratá-los como sendo unidimensional em apenas uma variável. Por exemplo, foi calculada a correlação entre uma variável criada da diferença entre os 12 valores em conjunto (DV) com cada uma das variáveis de Diferença de Valores separadas, o resultado indicou que, apesar de todas as correlações serem estatisticamente significativas, elas variaram de 0,575 entre DV5 e DV, até 0,162 entre DV8 e DV. Além disso, entre cada um dos valores apareceram correlações próximas de zero, como, por exemplo, entre DV4 e DV7, cuja correlação foi - 0,001. Isso demonstra que os valores não podem ser colocados em uma mesma variável por meio de medidas de distância, e as análises tem que ser feitas com os valores colocados separadamente no modelo.

Por fim, na criação da variável Semelhança das Ações Estratégicas, algumas das variáveis dicotômicas utilizadas apresentavam pouca variação na frequência das respostas, em algumas, quase todos os respondentes realizavam as ações; já em outras, quase nenhum realizava. Isso pode prejudicar a análise, pois reduz a variação 
REDES- Revista hispana para el análisis de redes sociales

Vol.22,\#5, Junio 2012

http://revista-redes. rediris.es

daquela que foi criada ao final. Novamente aqui o pesquisador terá que tomar cuidado, pois suas decisões podem interferir no resultado final das análises. Para exemplificar, foi rodado um modelo utilizando todas as 36 ações estratégicas apresentadas no questionário. Esse modelo apresentou $\mathrm{R}^{2}$ de 0,131 , mais baixo do que o Modelo 3 utilizado para fazer o teste das hipóteses. Ao colocar ações que não apresentavam variação entre os respondentes a medida de distância resultante também apresentava menor variação, influenciando o resultado da regressão. Além disso, acabam ocorrendo algumas alterações em quais das 12 variáveis DV ocorre a significância estatística. Por isso é importante fundamentar a escolha, teórica ou empiricamente, das variáveis que serão incluídas na transformação utilizando medidas de distância multivariadas.

A análise de Regressão Múltipla QAP está disponível no UCINET, bem como a sua versão mais recente, a Regressão Múltipla QAP (DSP) (DEKKER; KRACKHARDT; SNIDJERS, 2007), mas pode também ser realizada utilizando outros programas estatísticos, tais como o STATA, ou com a utilização de softwares específicos (MANLY, 2008). Todavia, essa técnica não é uma panaceia que pode ser utilizada indiscriminadamente.

Alguns limites precisam ser discutidos e são apresentados em trabalhos específicos que testam a robustez da técnica. O primeiro - e o mais importante, pois estará sempre por trás dos outros limites - é a baixa disseminação da técnica, em comparação com os testes estatísticos tradicionais, o que causa um certo limite nos testes de robustez feitos para a sua aplicação. Apesar de não ser um teste novo, pois foi apresentado inicialmente por Mantel em 1967 (MANLY, 2008), ele não é utilizado em larga escala. Sua utilização tem se ampliado a partir da aplicação na análise de redes e, com isso, os testes de robustez se ampliaram (KRACKHARDT, 1987; 1988; LEGENDRE, 2000; DEKKER; KRACKHARDT; SNIDJERS, 2007). Entretanto, como destacam Dekker, Krackhardt e Snidjers (2007), sua utilização deve ser parcimoniosa, e, no caso da necessidade de uma ampliação na sua utilização, novos testes devem ser realizados. Os maiores cuidados devem ser tomados quanto ao número de variáveis independentes a serem utilizadas na regressão em relação ao número de observações e a assimetria dos dados. Em ambos os casos pode haver degradação dos resultados especialmente pelo aumento da taxa de erro do tipo I (DEKKER; KRACKHARDT; SNIDJERS, 2007).

\section{Conclusões}


REDES- Revista hispana para el análisis de redes sociales

Vol.22,\#5, Junio 2012

http://revista-redes.rediris.es

Em alguns momentos a ARS tem sido criticada por ser um método sem uma teoria e que evoluiu muito na sua complexidade matemática e gráfica, mas que não gerou na mesma proporção conhecimento teórico. Quando Granovetter (1985) discute a importância dos relacionamentos, reavivando a Sociologia Econômica e trazendo para a ARS uma maior sustentação teórica, outra perspectiva foi aberta na área. É nesse mesmo espaço que novos avanços nas técnicas estatísticas, como o QAP, apresentado aqui, podem contribuir para as pesquisas em administração. É a partir da utilização de novos instrumentos que a ciência, em geral, e as ciências sociais, em particular, podem avançar. As pesquisas quantitativas em ciências sociais e ciências sociais aplicadas precisam se utilizar desses novos instrumentos para avançar nas teorizações sobre redes e relações sociais. Essa é a contribuição que este artigo procurou trazer ao apresentar a utilização de uma técnica nova e ainda pouco utilizada no país.

\section{Referências}

Ahuja, G. (2000), "Collaboration networks, structural holes, and innovation: a longitudinal study". Administrative Science Quarterly, v. 45, p. 425-455.

Bardin, L. (2009). Análise de conteúdo. Lisboa: Edições 70.

Bell, G.G.; Zaheer, A. (2007) "Geography, Network and Knowledge Flow". Organization Science, $18(6)$, p. 955-972.

Borgatti, S. P.; Foster, P. C. (2003). "The network paradigm in organizational research: a review and typology". Journal of Management, 29 (6), p. 991-1013.

Borgatti, S. P.; Everett, M. G. (1999), "Models core/periphery structures". Social Networks, v.21, p. 375-395.

Cohen, J. et al. (2003), Applied multiple regression/correlation analysis for the behavior sciences. 3 ed. Mahwah-NJ: LEA Publishers.

Coleman, J. S. (1988), "Social capital in the creation of human capital". American Journal of Sociology, v. 94, supplement, p. 95-120.

Cramer, D. (2003), Advanced quantitative data analysis. Maidenhead: Open University Press.

Dacin, M. T; Ventresca, M. J.; Beal, B. D. (1999), "The embeddedness of organizations: dialogue and directions". Journal of Management, 25 (3), p. 317-356. 
REDES- Revista hispana para el análisis de redes sociales

Vol.22,\#5, Junio 2012

http://revista-redes.rediris.es

Dekker, D.; Krackhardt, D.; Snijders, T. (2007), "Sensitivity of MRQAP Test to Collinearity and Autocorrelation Conditions". Psychometrika, 72 (4), p. 563-581.

Feld, S.L. (1982). "Social Structural Determinants of Similatities among Associates", American Sociological Review, 47 (6).

Granovetter, M. (1985), "Economic action and social structure: the problem of embeddedness". American Journal of Sociology, 91 (3), p.481-510.

Hanneman, A. R. (2001), Introduction to social network methods. Riverside: University of Califórnia.

Kirschbaum, C. (2006), "Renascença da indústria brasileira de filmes". Revista de Administração de Empresas. 46 (3).

Kirschbaum, C. (2011) "Rappers em São Paulo: conexões, desconexões e transgressões", CONLAB, consulta [15-12-2011] en <http://www.xiconlab.eventos.dype.com.br/resources/anais/3/1307751134 ARQUIVO Kirschbaum RappersemSaoPaulo XI-LABCS.pdf> .

Knoke, D; Yang, S. (2008), Social network analysis. 2. ed. Los Angeles: Sage.

Krackhardt, D. (1987), "QAP Partialling as a test of spuriousness". Social Network, v.9, p.171-186.

Krackhardt, D. (1988), "Predicting with networks: non-parametric multiple regression analysis of dyadic data". Social Network, v.10, p.359-381.

Kraskov, B.R.; Mouillot, D.; Shenbrot, G.I.; Khokhlova, I.S.; Poulin, R. (2010). "Deconstructing Spatial Patterns in Species Composition of Ectoparasite Communities", Global Ecology and Biogeography, 19 (4).

kraskov, B.R.; Mouillot, D.; Shenbrot, G.I.; Khokhlova, I.S.; Vinarski, M.V.; Korallo, N.P.; Poulin, R. (2010). "Similarity in Ectoparasite Faunas of Palaeartic rodents as a function of Host Phylogenetic, geography or environmental distances", International Journal for Parasitology, 40 (7).

Lengendre, P. (2000), "Comparison of permutation method for the partial correlation and partial mantel test". Journal of Statistical Computation and Simulation, 67 (1).

Lichstein, J.W. (2007). "Multiple Regression on Distance Matrices: a multivariate spartial analysis tool", Plant Ecology, 188(2). 
REDES- Revista hispana para el análisis de redes sociales

Vol.22,\#5, Junio 2012

http://revista-redes.rediris.es

Maciel, C.O. (2007). Práxis estratégica e imersão social em uma rede de organizações religiosas". 2007. Dissertação (Mestrado em Administração)-Setor de Ciências Sociais Aplicadas, Universidade Federal do Paraná, Curitiba.

Maciel, C.O.; Machado-da-Silva, C.L. (2009), "Práticas estratégicas em uma rede de congregações religiosas: valores e instituições, interdependência e reciprocidade." $R A P, 43$ (6).

Manly, B.J.F. (2008), Métodos estatísticos multivariados. 3. ed. Porto Alegre: Bookman.

Marques, E. (2003). Redes Sociais, Instituições e Atores Políticos no Governo da Cidade de São Paulo. São Paulo: Anna Blume.

Marques, E. (2010). "¿Como son las redes de los individuos en situación de pobreza en el Brasil urbano?", Redes. Revista Hispana para el Análisis de Redes Sociales, 18 (9). Consulta [22-02-2012] en <http://revista-redes.rediris.es>.

Mizruchi, M. S.; Marquis, C. (2006), "Egocentric, sociocentric, or dyadic: Identifying the appropriate level of analysis in the study of organizational networks". Social Networks, 28 (1), p. 187-208.

Murnieks, C.Y.; Haynie, J.M.; Wiltbank, R.E.; Harting, T. (2011) "'I Like How You Think': Similarity as an Interaction Bias in the Investor-Entrepreneur Dyad", Journal of Management Studies, 48 (7).

Powell, W. W.; Smith-Doerr, L. "Networks and economic life". (1994), In: SMELSER, N.J.; SWEDBERG, R. (Eds.). Handbook of Economic Sociology. Princeton: Russell Sage Foundation, 1994, p.368-402.

Scott, J. (2000), Social network analysis. 2. ed. London: Sage.

Simpson, W. QAP. (2011). The Quadratic Assignment Procedure. Disponível em: <http://www.stata.com/meeting/1nasug/simpson.pdf>. Acesso em: 24 abr. 2011.

Snijder, T. (2011). Statistical Models for Social Networks. Annual Review of Sociology, v.37, p. $131-153$.

SPSS. SPSS Tutorial. PASW Statistics 18, 2009.

Verdu, F.C.; Reinert, M. (2011) "Redes de relacionamentos interorganizacionais". In: EnANPAD, 35., 2011, Rio de Janeiro. Anais... Rio de Janeiro: ANPAD, 2011. 1 CD-ROM. 
REDES- Revista hispana para el análisis de redes sociales

Vol.22,\#5, Junio 2012

http://revista-redes. rediris.es

Verdu, F.C. (2010)"Redes de relacionamentos interorganizacionais, recursos e internacionalização: um estudo na cidade de Maringá (PR)". 2010. Tese (Doutorado em Administração)-Setor de Ciências Sociais Aplicadas, Universidade Federal do Paraná, Curitiba.

Wasserman, S.; Faust, K. (1994), Social network analysis. Cambridge: Cambridge University Press.

Zaheer, A.; Bell, G. G. (2005), "Benefiting from network position: firm capabilities, structural holes, and performance". Strategic Management Journal, v.26, p. 809-825. 
REDES- Revista hispana para el análisis de redes sociales

Vol.22,\#5, Junio 2012

http://revista-redes. rediris.es

\section{Apêndices}

Indicadores dos valores dos pastores pesquisados e instrumento de coleta dos dados relacionais

Fator: Inserção Política

A igreja evangélica deve aumentar sua participação na política nacional.

A igreja evangélica deve aumentar seu poder na política estadual/municipal.

A igreja evangélica deve dar mais apoio aos candidatos políticos.

Fator: Expansão

A igreja evangélica deve ter por princípio o crescimento da sua religião no Brasil.

A igreja evangélica deve fazer sua religião crescer além de outras religiões.

A igreja evangélica deve fazer com que a sua religião seja a que mais atrai fiéis.

Fator: Responsabilidade Social

Cabe à igreja evangélica a defesa de questões ambientais.

A igreja evangélica deve aumentar ainda mais a defesa de questões sociais.

As doações das congregações aos necessitados é uma obrigação dela na sociedade.

Fator: Modernidade

A igreja deve utilizar meios mais modernos de evangelização.

A igreja deve valorizar a informática como mecanismo de evangelização.

A igreja deve valorizar mais a internet como meio de evangelização.

Fator: Eficiência (Gestão)

As congregações devem utilizar mais mecanismos de controle na sua gestão.

As congregações devem fazer mais uso das formas de gestão das empresas.

A igreja deve empregar cada vez mais os princípios científicos de administração.

Fator: Customização

A igreja deve oferecer cultos de acordo com o "estilo" dos fiéis.

A igreja deve oferecer cultos temáticos de acordo com as necessidades dos fiéis.

A igreja deve fazer trabalhos mais dirigidos para públicos específicos.

Fator: Qualidade

As congregações devem se preocupar cada vez mais com a qualidade dos cultos.

A igreja deve se preocupar cada vez mais com a estética dos templos.

A igreja deve zelar cada vez mais pela qualidade dos músicos nos cultos.

Fator: Reputação

O cuidado com a reputação da igreja deve estar em primeiro lugar.

O cuidado com a reputação dos pastores deve estar em primeiro lugar.

Todos devem zelar pela reputação da igreja constantemente e acima de tudo.

Fator: Tradição

Os costumes dos fiéis da igreja não devem mudar.

A tradição da igreja é um dos seus maiores patrimônios.

A igreja deve se manter sempre em suas tradições.

Fator: Cooperação 
REDES- Revista hispana para el análisis de redes sociales

Vol.22,\#5, Junio 2012

http://revista-redes. rediris.es

A cooperação entre as congregações deveria aumentar cada vez mais.

As atividades conjuntas das congregações deveriam aumentar cada vez mais.

A igreja deve fazer aumentar a cooperação e ações entre as congregações.

Fator: Competição

Na realidade, as religiões precisam competir entre si para conseguir seus fiéis.

Igrejas evangélicas devem constantemente buscar fiéis de outras religiões e seitas.

Igrejas evangélicas devem competir com outras religiões para conseguir fiéis.

Fator: Mercado

As congregações devem aproveitar oportunidades de vender produtos evangélicos. As congregações devem comercializar mais produtos evangélicos aos seus fiéis.

Toda igreja evangélica deve aumentar a venda de produtos evangélicos.

Questão para coleta dos dados relacionais.

A seguir indique de três a seis nomes de dirigentes de outras congregações da sua igreja em Curitiba que você tem contato mais frequente.

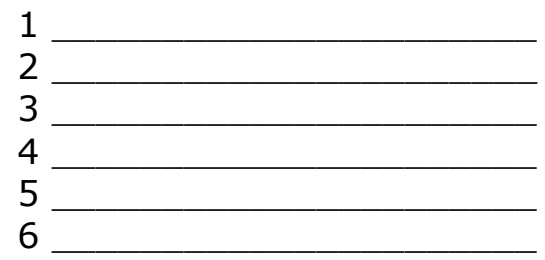

